\title{
Binary Nano-patterned Surfaces Prepared from Silane Monolayers
}

Antoine Pallandre, Karine Glinel ${ }^{\S}$, Alain M. Jonas* and Bernard Nysten

*correspondence to : jonas@poly.ucl.ac.be

\section{Supplementary Information}

Materials, reagents and solvents. Ethanol, toluene, chloroform, n-hexane and isopropanol (HPLC grade) were obtained from Acros Organics. Sulfuric acid (98\%) and hydrogen peroxide $(30 \%)$ were from Merck VWR. The anionic PMMA resist $\left(\mathrm{M}_{\mathrm{n}}=142000 \mathrm{~g} \cdot \mathrm{mol}^{-1}\right.$; $M_{w}=160000$ g.mol${ }^{-1}$ ) was obtained from Polymer Source (ref. P1409); for spin-coating, it was dissolved into toluene $(20 \mathrm{~g} / \mathrm{ml}$ ), filtered onto PVDF filters (Millipore, pore size $0.22 \mu \mathrm{m}$ ), and stored in a closed vessel. All silanes (Scheme 1), obtained from Gelest, were stored in flasks under anhydrous argon and used as received. Poly(diallylammonium chloride) (PDADAMC, $M_{w}=100000-200000$ g.mol ${ }^{-1}$ ) and poly(styrene sulfonate) (PSS, $M_{w}$ about $70000 \mathrm{~g} \cdot \mathrm{mol}^{-1}$ ) were purchased from Aldrich and used without purification.

One-side polished $475 \mu \mathrm{m}$-thick silicon wafers were obtained from ACM (Applications Couches Minces, France) with (100) orientation; they were cut into squares of $1.5 \mathrm{~cm}$ side. The wafers were cleaned for 20 min in freshly prepared piranha solutions $\left(\mathrm{H}_{2} \mathrm{O}_{2}(27 \%)\right.$ and $\mathrm{H}_{2} \mathrm{SO}_{4}(98 \%) 1: 1$ v/v. Caution: piranha solutions react violently with organic materials and should not be stored in closed containers), then abundantly rinsed with water purified with a Millipore purification system (resistivity lower than 18.2 M $\Omega . \mathrm{cm}$ ). Wafers were dried by spinning with a spin-coater, then placed for 4 hours at $70^{\circ} \mathrm{C}$ under a primary vacuum. The thickness of the native oxide layer lying on the silicon was obtained by ellipsometry and was found to be $1.3 \mathrm{~nm}$, with a standard deviation of $0.3 \mathrm{~nm}^{1}$

Gas-phase silanation. A glass reactor was designed to conduct anhydrous gas phase silanation. It consists in a jacketed round bottom vessel, fitted with a cover with two feedthroughs with poly(tetrafluoroethylene) stopcocks (gas inlet and vacuum outlet). A third, 
unstoppered, feedthrough was used to record temperature. A Viton® seal was used to seal the reactor. A fourth stoppered inlet, directly connected to the side of the reactor, allowed to inject the silane directly at the bottom of the reactor. The temperature in the reactor was controlled by flowing silicone oil through the jacket.

Before any silanation, all glassware was cleaned by immersion for one night in a $\mathrm{KOH}$-saturated isopropanol bath, and rinsed thoroughly with water. The closed reactor was then dried at $100^{\circ} \mathrm{C}$ for 12 hours under continuous pumping (primary vacuum ensured by a rotary pump fitted with a liquid nitrogen cold trap). After introduction of dry argon (grade 5.5 from Air Products), about 12 freshly cleaned wafers were placed over a two-stories glass tray fitted with an upper cover protecting samples from contamination resulting from silane condensation on the reactor cover, and introduced in the reactor. The samples and reactor were annealed at $70^{\circ} \mathrm{C}$ for 4 hours under primary vacuum. Dry ultrapure argon was then introduced and the silane was injected. The reaction was allowed to proceed for $24 \mathrm{~h}$ in a slight overpressure of dry argon. After $24 \mathrm{~h}$, the reactor was pumped until complete removal of remaining traces of silane, opened in a flow of dry argon. The silanized wafers were then washed in a Soxhlet (chloroform or acetone depending on the presence of a PMMA mask or not) for $24 \mathrm{~h}$.

Electron beam nanolithography (EBL). All processes, including wafer cleaning, were conducted in a class 1000 clean room environment. PMMA was spun-coated onto the wafers to obtain films about $95 \mathrm{~nm}$ thick, which were subsequently annealed at $170^{\circ} \mathrm{C}$ for 12 hours. EBL was performed with a XL 30 S Philips scanning electron microscope (field emission gun) associated with an Elphy Plus lithographic system. First, 100 square frames of $42 \mu \mathrm{m}$ side and $500 \mathrm{~nm}$ line thickness were drawn over the surface, developed, and etched in the silicon by anisotropic $\mathrm{SF}_{6}$ plasma. These frames, which can easily be detected by visual inspection or with an optical microscope, later served to locate more easily the nanopatterns. After wafer cleaning, a new PMMA film was deposited and annealed, and EBL was performed in each $42 \times 42 \mu \mathrm{m}^{2}$ frame according to different settings and parameters. The size of writing fields was $50 \times 50 \mu \mathrm{m}^{2}$ 
with $5 \mathrm{~mm}$ working distance between sample and the lens; the electron beam was focussed through the observation of so-called contamination dots. Dwelltime parameter was adjusted during the lithography depending on the expected size of the pattern. The development of the exposed regions was performed with a mixture of isopropanol and methyl isobutylketone (1:3 $\mathrm{v} / \mathrm{v}$ ) for $90 \mathrm{~s}$. The masks were then rinsed in isopropanol for $30 \mathrm{~s}$ and in ultra pure water for $5 \mathrm{~min}$. The wafers were finally exposed for $30 \mathrm{~s}$ to an oxygen plasma at $50 \mathrm{~W}$ (in a Technics Plasma Gmbh, 300 Plasma system) to remove residual contaminants in developed regions. Two samples were always systematically exposed identically; one of them was used to check the quality of the lithography, using a $7 \mathrm{~nm}$ titanium lift-off.

Fabrication of chemical nanopatterns. Electron-beam nanolithographied masks were placed in the silanation reactor directly after fabrication, and a first silane was injected as described above. After $24 \mathrm{~h}$ reaction, the wafers were extracted with acetone for $24 \mathrm{~h}$ in a Soxhlet, in order to remove the PMMA mask and traces of unreacted silane. The wafers were then directly placed in the silanation reactor, and a second silanation was performed as described above.

Oxidation of 7-octenyl-trichloro-silane (1) monolayers. The oxidation was performed in agreement with the method proposed by Wasserman et al. $^{2}$ The 7 -octenyl-trichloro-silanegrafted wafers were placed in a reaction mixture consisting of $\mathrm{KMn}_{4} \mathrm{O}(0.5 \mathrm{mM}), \mathrm{K}_{2} \mathrm{CO}_{3}(1.8$ $\mathrm{mM}$ ) and $\mathrm{NaIO}_{4}(19.5 \mathrm{mM})$ in $100 \mathrm{~mL}$ water (MilliQ). After $12 \mathrm{~h}$ reaction at room temperature, they were rinsed with $\mathrm{NaHSO}_{3}(0.3 \mathrm{M})$, water, $0.1 \mathrm{M} \mathrm{HCl}$, water and ethanol, to remove the oxidant. The surface of the reacted monolayer was sometimes found to be covered by a thin white deposit from the reagent solution. Different methods were tested to remove this deposit. It was found that mechanical removal by gentle scrapping in ethanol with a poly(tetrafluoroethylene) spatula gave the best results. XRR and XPS performed on so-cleaned monolayers confirmed that the treatment did not affect the monolayer quality, while effectively removing most traces of contaminants. 
Controlled deposition of polyelectrolyte multilayers. Polyelectrolyte multilayers were deposited by alternate dipping of the substrates in aqueous solutions of polycation and polyanion (10 $\mathrm{mM}$ in monomer unit, with no salt added). Three rinsing steps in purified water and one drying step were applied in-between each adsorption step. This process was cycled five times.

\section{Instrumental techniques.}

X-ray reflectometry (XRR). The experimental setup is based on a Siemens D5000 2-circles goniometer of $30 \mathrm{~cm}$ radius and $0.002^{\circ}$ positioning accuracy. X-rays of $1.5418 \AA$ wavelength $(\mathrm{Cu} \mathrm{K} \alpha)$ were obtained from a Siemens rotating anode operated at $40 \mathrm{kV}$ and $300 \mathrm{~mA}$, fitted with a graphite secondary monochromator and a scintillation counter. The beam was defined by a $40 \mu \mathrm{m}$-wide slit placed at $17.5 \mathrm{~cm}$ from the focal spot. Parasitic scattering was decreased by a $200 \mu \mathrm{m}$ wide slit placed after the $40 \mu \mathrm{m}$ slit. The sample was placed at the center of the goniometer with an automated procedure, using a vertical stage of $1 \mu \mathrm{m}$ resolution. The intensity was scaled to unit incident intensity, then corrected for spill-over at very low angles of incidence. Data are reported as a function of $\mathrm{k}_{\mathrm{zo}}$, the vertical component of the wavevector of the incident photons in a vacuum.

XRR data were analyzed in two ways. First, a Patterson function was computed from the data as described elsewhere, ${ }^{1}$ and the average thickness of the monolayer was obtained from the position of the subsidiary correlation peak in this function. Second, a model of electron density was fitted to the data. The electron density profile was discretized as a succession of flat slabs of width $\Delta=0.5 \mathrm{~nm}$, approximately corresponding to the resolution of the experiments defined by $\mathrm{k}_{\mathrm{z} 0 \text {, max }}$, the maximum value of $\mathrm{k}_{\mathrm{z} 0}$ for which the signal is not yet dominated by the background $\left(\Delta=\pi /\left(2 . k_{z 0, \max }\right)\right)$. The number of slabs required was initially selected from the known thickness of the monolayer determined from the Patterson function. It was progressively incremented to take into account the width of the interfaces, until the fit was deemed satisfactory as judged from 
the value of chi-square obtained. The fit parameters were the height (electron density) of each slab, a general scaling parameter, and a constant background value. The reflectivity was computed using Parratt's formalism. ${ }^{3,4}$ To ease interpretation, the electron density profiles are represented by dots placed at the centers of each slab; cubic spline interpolation of these profiles was used to draw a smooth line through the dots.

X-ray Photoelectron spectroscopy (XPS). XPS spectra were obtained with a Scienta ESCA 300 spectrometer, using an Al Ka line source $(\mathrm{h} v=1486 \mathrm{eV})$ with a pass energy of $75 \mathrm{eV}$ and a resolution of $0.6 \mathrm{eV}$. The typical operating pressure was $10^{-10}$ Torr. The energy calibration was performed by setting the $\mathrm{C} 1 \mathrm{~s}$ peak to $284.6 \mathrm{eV}$ binding energy.

Atomic Force Microscopy (AFM). AFM images were recorded in air with an Autoprobe CP from Thermomicroscopes using either a $100 \mu \mathrm{m}$ or a $5 \mu \mathrm{m}$ scanner. The images were recorded either in contact mode (topography and lateral force, C-AFM and LFM) or in intermittent-contact mode (IC-AFM), as indicated in the text. For C-AFM and LFM, the cantilevers were silicon Ultralevers $^{\mathrm{TM}}$ (Thermomicroscopes) with a spring constant of about $0.25 \mathrm{~N} / \mathrm{m}$ and an integrated Si tip with a typical radius of curvature of $10 \mathrm{~nm}$. For IC-AFM, Pointprobe ${ }^{\circledR}$ Tapping $^{\mathrm{TM}}$-mode sensors (Nanosensors) were used. These cantilevers had resonance frequency around 160 $165 \mathrm{kHz}$, a typical spring constant of about $40 \mathrm{~N} / \mathrm{m}$ and an integrated Si tip with a radius around $10 \mathrm{~nm}$. Only a second order flattening procedure (line by line) was performed on AFM images.

Contact angle measurements. Water contact angles were measured under ambient atmosphere at $20^{\circ} \mathrm{C}$, using the sessile drop method and an image analysis of the drop profile with a home made system. The water (MilliQ) droplet volume was $0.5 \mu \mathrm{L}$. 
Structural data on silane monolayers obtained by gas phase silanation

\begin{tabular}{|c|c|c|c|}
\hline Compound & $d_{X R R}(n m){ }^{(a)}$ & $L_{\text {theo }}(n m)^{(b)}$ & $\theta_{\mathrm{H} 2 \mathrm{O}}\left({ }^{\circ}\right)^{(\mathrm{c})}$ \\
\hline 1 & 1.05 & 1.08 & $98.6 \pm 0.2$ \\
\hline oxidized 1 & 0.85 & 0.99 & $70 \pm 5$ \\
\hline 2 & 0.95 & 1.47 & $71.3 \pm 0.9$ \\
\hline 3 & 0.95 & 1.32 & $105.7 \pm 0.3$ \\
\hline 4 & 0.85 & 1.32 & $104.5 \pm 0.8$ \\
\hline 5 & 1.10 & 1.30 & $115 \pm 0.5$ \\
\hline 6 & 1.15 & 1.30 & $113.2 \pm 0.8$ \\
\hline 7 & gel formation & 0.45 & $73.1 \pm 0.8$ \\
\hline 8 & gel formation & 0.89 & $78.3 \pm 1$ \\
\hline 9 & gel formation & 0.53 & $67.2 \pm 0.8$ \\
\hline 10 & 0.7 & 0.53 & $54.6 \pm 1.1$ \\
\hline 11 & gel formation & 0.90 & $72 \pm 0.7$ \\
\hline 12 & 0.7 & 0.84 & $83.4 \pm 0.4$ \\
\hline 13 & 0.9 & 0.70 & $80.4 \pm 0.5$ \\
\hline
\end{tabular}

Table 1. Structural data on silane monolayers obtained by gas phase silanation.

(a) experimental thickness of the monolayer obtained by X-ray reflectometry (estimated error: $0.1 \mathrm{~nm}$; roughness of the Si wafers: about $0.3 \mathrm{~nm}$ )

(b) computed extended length of the organic part of the molecule (distance between the first carbon atom and the farthest atom of the chain in all-trans conformation; the Van der Waals radius of the farthest atom was added to obtain the extended length). 


\section{(c) water static contact angle.}

\section{Data on the removal of the PMMA resist prior to silanation.}

The success of the silanation reactions critically rests on the complete elimination of residual PMMA fragments in exposed and developed regions prior to the first silanation, and of residual PMMA chains in unexposed regions prior to the second silanation. To fully eliminate PMMA fragments in exposed zones after development, an oxygen plasma was applied for 30 sec after development (see above). This effectively destroys any thin layer of organic material remaining at the bottom of lithographied holes after development.

However, this cleaning process cannot be applied prior to the second silanation, since it would simultaneously destroy previously silanized regions of the sample. Therefore, we investigated whether a simple Soxhlet extraction could successfully remove unexposed PMMA on Si wafers. A silicon wafer was first cleaned in a piranha solution, and immediately analyzed by XRR. A PMMA film was then directly spun-coated onto the wafer, and annealed overnight at $160^{\circ} \mathrm{C}$ to relax the film. The wafer was subsequently placed in an acetone Soxhlet, and the PMMA film was extracted for $24 \mathrm{~h}$. The XRR of the sample was then measured again. Figure 1 compares the reflectograms and electron density profiles for the silicon wafer measured before spincoating, and after PMMA extraction. The profiles and reflectograms are virtually identical, testifying for the complete removal of the PMMA from the wafer after the acetone Soxhlet.

In addition, XRR was measured on a sample silanized with 6, and on a patterned sample (dots and lines of $\mathbf{1}$ in a background of $\mathbf{6}$ ). The patterned region was small enough to be negligible as compared to the footprint of the X-ray beam on the sample, which implied that the experiment was essentially sensitive to the silanized background. The two measurements, together with the corresponding electron density profiles, are presented in Fig.1. Again, both samples are virtually identical, indicating successful silanization after PMMA extraction. 

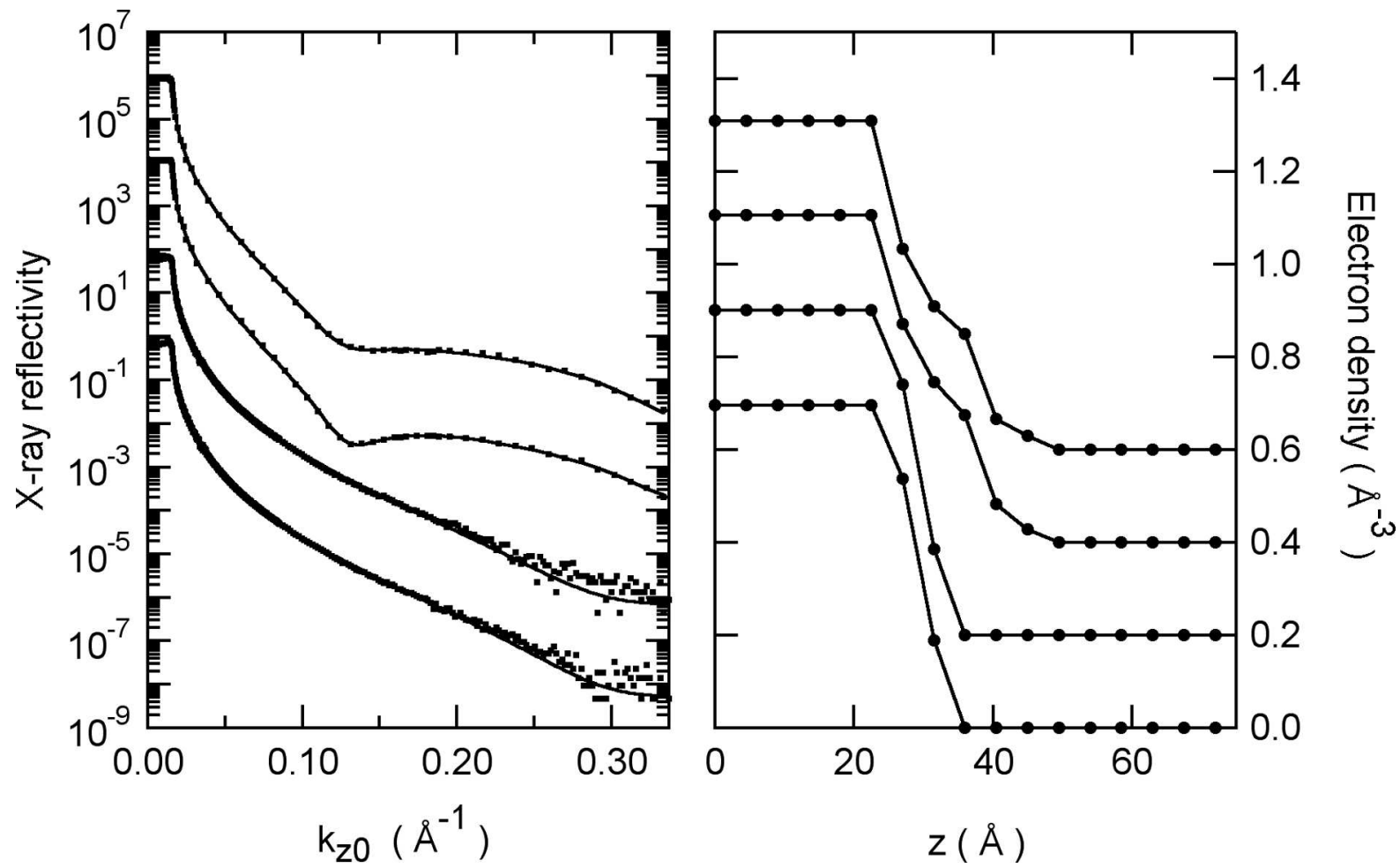

Figure 1. Left. X-ray reflectograms (square: experimental data; continuous lines: fits) of, from bottom to top, (1) a bare silicon wafer measured just after piranha cleaning, (2) the same wafer after deposition of a PMMA film subsequently removed by an acetone Soxhlet, (3) a Si wafer silanized with $\mathbf{6}$, and (4) a Si wafer silanized with $\mathbf{6}$ after deposition and removal by Soxhlet extraction of a PMMA film. Successive curves are multiplied by $10^{2 n}$, where $n$ is an integer between 0 and 3 . Right. Electron density profiles obtained from the fits of the reflectivity data. Curves have been displaced vertically by integral multiples of $0.2 \AA^{-3}$, and are in the same order as in the left part.

\section{References}

[1] Arys, X.; Laschewsky, A.; Jonas, A. M. Macromolecules, 2001, 34, 3318.

[2] Wasserman, S. R.; Whitesides, G. M.; Tidswell, I. M.; Ocko, B. M.; Pershan, P. S.; Axe, J. D. J. Am. Chem. Soc. 1989, 111, 5852.

[3] Parratt, L. G. Phys. Rev. 1954, 95, 359.

[4] Parratt, L. G. J. Chem. Phys. 1956, 53, 597. 
